\title{
ON HYPERSURFACES IN A LOCALLY AFFINE RIEMANNIAN BANACH MANIFOLD
}

\section{EL-SAID R. LASHIN and TAREK F. MERSAL}

Received 12 September 2001

\begin{abstract}
We prove that an essential hypersurface of second order in an infinite-dimensional locally affine Riemannian Banach manifold is a Riemannian manifold of constant nonzero curvature.
\end{abstract}

2000 Mathematics Subject Classification: 53C20, 53C40.

1. Introduction. Let $M$ be an infinite-dimensional Banach manifold of class $C^{k}, k \geq$ 1 , modelled on a Banach space $E$, and let $\bar{g}^{1}$ be a symmetric bilinear form defined on $M$, that is, $\overline{\mathfrak{g}}^{1} \in L_{2}(M ; \mathbb{R})$.

DEFINITION 1.1 [2]. A metric $\bar{g}^{1}$ is said to be a strongly nonsingular, if $\bar{g}^{1}$ associates a mapping

$$
\overline{\mathfrak{g}}^{1 *}: x \in M \longrightarrow \overline{\mathfrak{g}}_{x}^{1 *}=\bar{g}^{1}(x, \cdot) \in L(M ; \mathbb{R}) \equiv M^{*},
$$

which is bijective. Furthermore, $M^{*}$ is the dual space of $M$.

Now, let $\bar{\Gamma}$ be the linear connection on $M$.

DEFINITION 1.2 [2]. A differentiable Banach manifold $(M, \bar{\Gamma})$ of class $C^{k}, k \geq 3$, is called locally affine if their curvature and torsion tensors are zero.

In general, it is proved in [2] that, a Banach manifold $(M, \bar{\Gamma})$ is locally affine if and only if there exists an atlas $\mathscr{A}$ on $M$, such that for any chart $c \in \mathscr{A}, \Gamma \equiv 0$, where $\Gamma$ is the model of the linear connection $\bar{\Gamma}$.

DEFINITION 1.3 [2]. In a space $M$ with a first fundamental form $\varphi=\bar{g}_{x}^{1}\left(\bar{X}_{1}, \bar{X}_{2}\right)$, the hypersurface $N \subset M$, which is defined by the equation

$$
\overline{\mathcal{g}}_{x}^{1}(\bar{X}, \bar{X})=e r^{2}, \quad e= \pm 1,0 \neq r \in \mathbb{R},
$$

is called an essential hypersurface of the second order in the space $M$.

2. Essential hypersurface in a locally affine Banach manifold. Let $M$ be a locally affine Banach manifold, and assume that $\bar{g}^{1}$ is a strongly nonsingular metric on $M$, then the pair $\left(M, \bar{g}^{1}\right)$ is a Riemannian Banach manifold. Let $N$ be a subset of $M$ such that

$$
N=\left\{\bar{x} \in M: \bar{g}_{x}^{1}(\bar{x}, \bar{x})=e r^{2}, 0 \neq r \in \mathbb{R}, e= \pm 1\right\},
$$

that is, $N$ is an essential hypersurface in $M$. Also, we can define $N$ as follows:

$$
N=\left\{\bar{x} \in M: F(\bar{x})=\bar{g}_{x}^{1}(\bar{x}, \bar{x})-e r^{2}=0\right\},
$$


where $F: M \rightarrow \mathbb{R}$ is a differentiable function, such that for all $\bar{x} \in N, 0 \neq D F_{\bar{x}} \in$ $L\left(T_{\bar{x}} M ; \mathbb{R}\right)$ is a linear mapping on the tangent space $T_{\bar{x}} M$.

Since $D F_{\bar{x}}(\bar{Y})=2 \bar{g}_{x}^{1}(\bar{x}, \bar{Y})$ for all $\bar{x} \in N$, and $\bar{g}^{1}$ is nonsingular, then there exists a vector $\bar{Y} \in T_{\bar{x}} M$ such that $\bar{g}_{x}^{1}(\bar{x}, \bar{Y}) \neq 0$, and consequently $D F_{\bar{x}} \neq 0$. Hence, $N$ will be a closed submanifold of $M$.

Denoting by $\bar{i}: \bar{x} \in N \rightarrow \bar{i}(\bar{x})=\bar{x} \in M$ the inclusion mapping.

Let $c=(U, \Phi, E)$ be a chart at $\bar{x} \in M$, and let $d=(V, \Psi, F \subseteq E)$ be a chart at $\bar{x} \in N$, where the Banach spaces $E$ and $F$ are the models of the manifolds $M$ and $N$ with respect to the charts $c$ and $d$, respectively. Furthermore, we have that $\Psi(\bar{x})=x$ is the model of the point $\bar{x}$ with respect to the chart $d, z=\Phi(\bar{x})$ is the model of $\bar{x}$ with respect to the chart $c$, and $i$ is the model of $\bar{i}$ with respect to the charts $c$ and $d$. Then, we obtain that

$$
i: x=\Psi(\bar{x}) \in \Psi(V) \subset F \longrightarrow i(x)=z=\Phi(\bar{x}) \in \Phi(V) \subset E .
$$

In this case, (2.3) is called the local equation of the submanifold $N \subset M$ with respect to the charts $c$ and $d$. Also, $N$ will be a Riemannian submanifold of $M$ with induced metric $\bar{g}^{2}$, which is defined by the rule

$$
\overline{\mathfrak{g}}_{x}^{2}\left(\bar{X}_{1}, \bar{X}_{2}\right)=\overline{\mathfrak{g}}_{i(x)}^{1}\left(T_{x} i\left(\bar{X}_{1}\right), T_{x} i\left(\bar{X}_{2}\right)\right),
$$

for all $\bar{x} \in N$ and $\bar{X}_{1}, \bar{X}_{2} \in T_{\bar{x}} N$, where $T_{\bar{x}} \bar{i}: T_{\bar{x}} N \rightarrow T_{\bar{x}} M$ is the tangent mapping of $\bar{i}$ at the point $\bar{x} \in N$ (see [1]).

Assume that $\bar{g}^{2}$ is strongly nonsingular metric on $N$. Also we have that, $M$ and $N$ are Riemannian manifolds with free-torsion connections $\bar{\Gamma}^{1}, \bar{\Gamma}^{2}$, respectively such that $\bar{\nabla}^{1} \bar{g}^{1}=0$ and $\bar{\nabla}^{2} \bar{g}^{2}=0$ (see [3, 4]). Let $X_{1}, X_{2} \in F$ be the models of $\bar{X}_{1}, \bar{X}_{2} \in T_{\bar{x}} N$ with respect to the chart $d$ on $N$. Then $Y_{1}=D i_{x}\left(X_{1}\right), Y_{2}=D i_{x}\left(X_{2}\right)$ are the models of $\bar{X}_{1}$, $\bar{X}_{2}$ with respect to the chart $c$ on $M$.

In this case, the local equation of (2.4) takes the form

$$
g_{x}^{2}\left(X_{1}, X_{2}\right)=g_{x}^{1}\left(D i_{x}\left(X_{1}\right), D i_{x}\left(X_{2}\right)\right) .
$$

Now, we are ready to introduce our main theorem.

THEOREM 2.1. An essential hypersurface of order two in an infinite-dimensional locally affine Riemannian Banach manifold is a Riemannian manifold of constant nonzero curvature.

Proof. Let $N \subset M$ be an essential hypersurface of order two in a Banach manifold $M$. Then, for any arbitrary charts $c=(U, \Phi, E)$ on $M$ at the point $\bar{x} \in N$, and $d=$ $(V, \Psi, F)$ on $N$ at the same point $\bar{x}$, the equation of the submanifold $N$ takes the form

$$
g_{x}^{1}(y, y)=e r^{2}
$$

This means that $y=i(x), x \in \Psi(V)$ is the model of $\bar{x} \in N$ with respect to the chart $d$. In other words, the equation of the submanifold $N$, with respect to the charts $c$ and $d$, can be rewritten as follows:

$$
g_{x}^{1}(i(x), i(x))=e r^{2},
$$


for all $0 \neq r \in \mathbb{R}$, and $x \in \Psi(V), e= \pm 1$. Differentiating (2.7) with respect to $x$ in the direction $X \in F$, we obtain

$$
g_{x}^{1}\left(i(x), D i_{x}(X)\right)=0, \quad \forall x \in \Psi(V) \subset F, X \in F .
$$

Once again differentiating (2.8) with respect to $x$ in the direction $Y \in F$ we obtain

$$
g_{x}^{1}\left(D i_{x}(Y), D i_{x}(X)\right)+g_{x}^{1}\left(i(x), D^{2} i_{x}(Y ; X)\right)=0 .
$$

By using (2.5) and (2.8) in the above equation, we obtain

$$
g_{x}^{2}(X, Y)=-g_{x}^{1}\left(i(x), D^{2} i_{x}(Y ; X)-D i_{x}\left(\Gamma_{x}^{2}(X, Y)\right)\right),
$$

where $\Gamma^{2}$ is the model of the linear connection $\bar{\Gamma}^{2}$ with respect to the chart $d$ on $N$, that is,

$$
g_{x}^{1}\left(i(x), \nabla^{2} D i_{x}(Y ; X)\right)=-g_{x}^{2}(X, Y),
$$

for all $x \in \Psi(V) \subset F$ and all $X, Y \in F$, where $\nabla^{2}$ is the covariant differentiation corresponding to $\Gamma^{2}$ on $N$.

Now, the first differential equation of the hypersurface $N \subset M$ takes the form (see [5])

$$
\nabla^{2} D i_{x}(X, Y)=e A_{x}(X, Y) \xi_{x}
$$

where $\bar{\xi}_{x} \in T_{0}^{1}(M)$ is a unit vector in $M$ orthogonal to $N$ at the point $\bar{x} \in M$, that is,

$$
\bar{g}^{1}\left(\bar{\xi}_{x}, \bar{\xi}_{x}\right)=e, \quad \bar{g}^{1}\left(\bar{\xi}_{x}, \bar{X}\right)=0,
$$

for all $\bar{x} \in N \subset M$, and for all $\bar{X} \in T_{x} N$, and $A_{x}$ is the second fundamental form for the hypersurface $N$, such that

$$
A_{x}(X, Y)=g_{x}^{1}\left(D^{2} i_{x}(X, Y), \xi_{x}\right)=-g_{x}^{1}\left(D i_{x}(X), D \xi_{x}(Y)\right) .
$$

Taking into account that $T_{x} \bar{i} \in T_{0}^{1}(N), \bar{\xi}_{x} \in T_{0}^{1}(M)$, and (2.12) we conclude that $A_{x}$ is a symmetric tensor of type $(0,2)$ on $N$ at the point $\bar{x} \in N$.

Now let $\xi: x=\Psi(\bar{x}) \in \Psi(V) \subset F \rightarrow \xi_{x} \in E$ be the model of the vector field

$$
\bar{\xi}: \bar{x} \in N \longrightarrow \bar{\xi}_{\bar{x}} \in T_{\bar{x}} M,
$$

with respect to the charts $c$ and $d$ at the point $\bar{x}$. Then the local equation of equality (2.13) takes the form

$$
g^{1}\left(\xi_{x}, \xi_{x}\right)=e, \quad g^{1}\left(D i_{x}(X), \xi_{x}\right)=0
$$

for all $x \in \Psi(V) \subset F$ and for all $X \in F$.

Now from (2.8) and (2.16), we deduce that the unit normal vector $\xi_{\bar{x}}$ on $N$ can be defined as follows:

$$
\xi_{x}=\lambda \dot{i}(x) ; \quad \lambda \in \mathbb{R} .
$$


Also from (2.7) and the first equality of (2.16) and using (2.17), we obtain

$$
g_{x}^{1}(\lambda \dot{i}(x), \lambda \dot{i}(x))=e,
$$

that is,

$$
\lambda^{2} g_{x}^{1}(i(x), i(x))=\lambda^{2}\left(e r^{2}\right)=e,
$$

which implies that $\lambda= \pm 1 / r$. It is convenient to take $\lambda=+1 / r$. Then

$$
\xi_{x}=\left(\frac{1}{r}\right) i(x)
$$

Substituting by (2.7), (2.12), and (2.20) in (2.11), we obtain

$$
g_{x}^{1}\left(i(x), e A_{x}(X, Y) \dot{\xi}_{x}\right)=-g_{x}^{2}(X, Y)
$$

which implies that

$$
A_{x}(X, Y)=-\frac{1}{r} g_{x}^{2}(X, Y) .
$$

From the above we conclude that, the first and the second fundamental forms for the essential hypersurface of the second order are proportional. Now from (2.12), (2.20), and (2.22), we obtain

$$
\nabla_{2} D i_{x}(X, Y)=e A_{x}(X, Y) \xi_{x}=-\frac{e}{r^{2}} g_{x}^{2}(X, Y) \dot{i}(x) .
$$

Putting $\kappa_{0}=e / r^{2}$, then

$$
\nabla^{2} D i_{x}(X, Y)+\kappa_{0} g_{x}^{2}(X, Y) \dot{i}(x)=0 .
$$

Now we find that, the integral condition of the first differential equation of the hypersurface is (see [5])

$$
g_{x}^{2}\left(R_{x}^{2}(Y ; Z, X), S\right)=e A_{x}(\underline{Z}, Y) A_{x}(\underline{X}, S) .
$$

REMARK 2.2. In Formula (2.25), there exists an alternation with respect to the underlined vectors without division by 2 . This convention will be used henceforth.

If we use (2.22), the integral condition for (2.12), will take the form

$$
g_{x}^{2}\left(R_{x}^{2}(Y ; Z, X), S\right)=\frac{e}{r^{2}} g_{x}^{2}(\underline{Z}, Y) g_{x}^{2}(\underline{X}, S) .
$$

Using $\kappa_{0}=e / r^{2}$, we obtain

$$
g_{x}^{2}\left(S, R_{x}^{2}(Y ; Z, X)-\kappa_{0} g_{x}^{2}(\underline{Z}, Y) \underline{X}\right)=0, \quad \forall S \in F .
$$

Taking into account that $g_{x}^{2}$ is nonsingular, we obtain

$$
R_{x}^{2}(Y ; Z, X)=\kappa_{0} g_{x}^{2}(\underline{Z}, Y) \underline{X},
$$

where $\bar{R}_{x}^{2}$ is the curvature tensor of the submanifold $N \subset M$. Hence, from (2.28) we deduce that the essential hypersurface (hypersphere or pseudohypersphere) of the second order in a locally affine infinite-dimensional Banach manifold $M$ is a Riemannian manifold of constant nonzero curvature $\kappa_{0}$, and this completes the proof. 


\section{REFERENCES}

[1] V. E. Fomin, Differential'nay Geometries, Differential calculus. Manifolds. Linear connection, Kazoo University, Kazoo, 1983 (Russian).

[2] _ Methods and Indications to a Special Course in Differential Geometry of Banach Manifolds, Kazan University, Kazan, 1986.

[3] S. Kobayashi and K. Nomizu, Foundations of Differential Geometry. Vol I, John Wiley \& Sons, New York, 1963.

[4]___ Foundations of Differential Geometry. Vol. II, John Wiley \& Sons, New York, 1969.

[5] E. R. Lashin, On hypersurfaces in Banach manifolds, J. Faculty of Education, Ain-Shams Univ. 20 (1995), 257-267.

EL-Said R. Lashin: Department of Mathematics, Faculty of Science, Menoufiya UniVERSITY, MENOUFIYA 32511, EGYPT

Tarek F. Mersal: Department of Mathematics, Faculty of Science, Menoufiya UniVERSITY, MENOUFIYA 32511, EgYPT

E-mail address: tmersa1@yahoo.com 


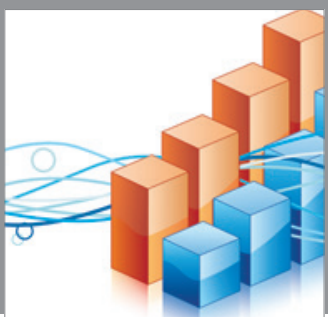

Advances in

Operations Research

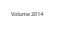

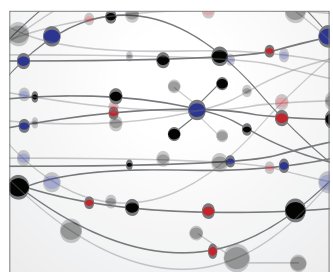

\section{The Scientific} World Journal
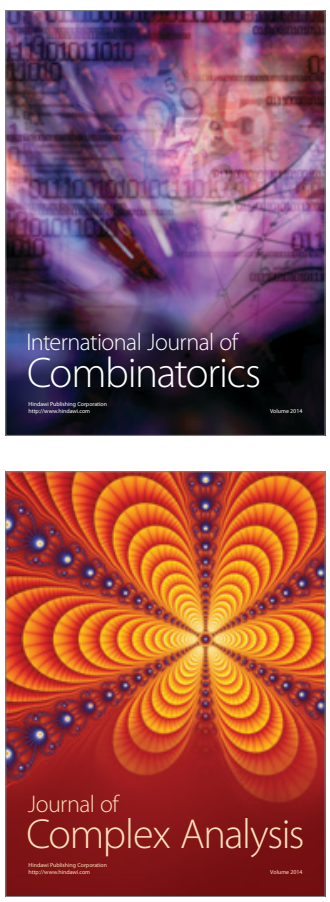

International Journal of

Mathematics and

Mathematical

Sciences
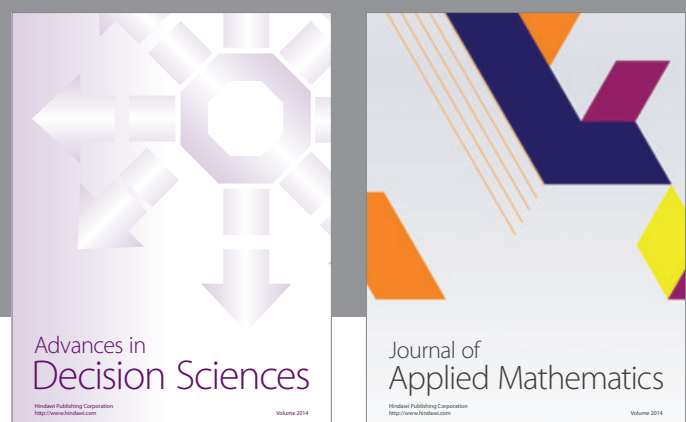

Journal of

Applied Mathematics
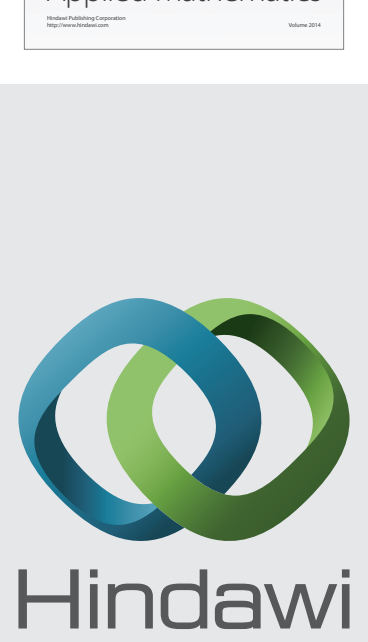

Submit your manuscripts at http://www.hindawi.com
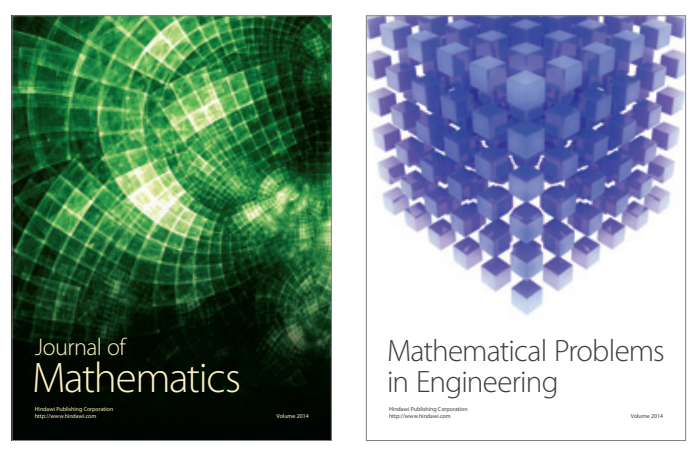

Mathematical Problems in Engineering
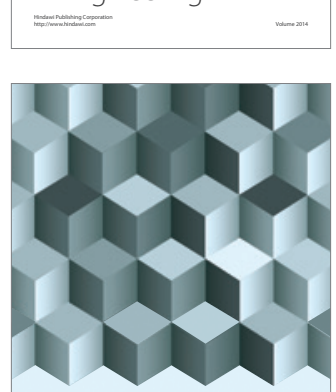

Journal of

Function Spaces
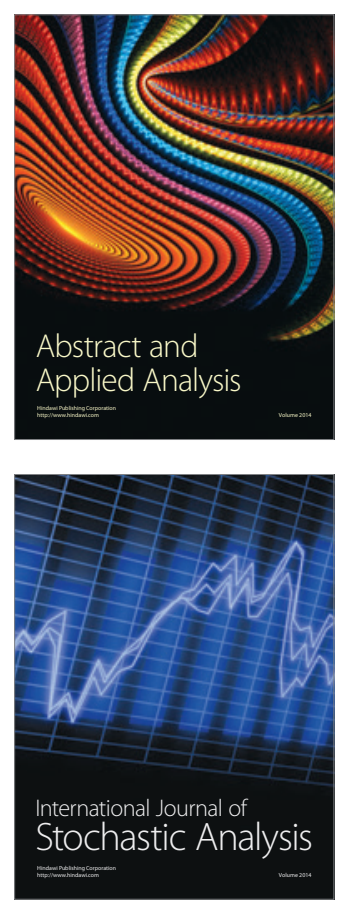

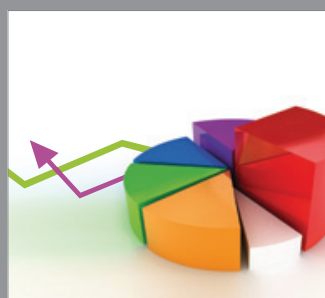

ournal of

Probability and Statistics

Promensencen
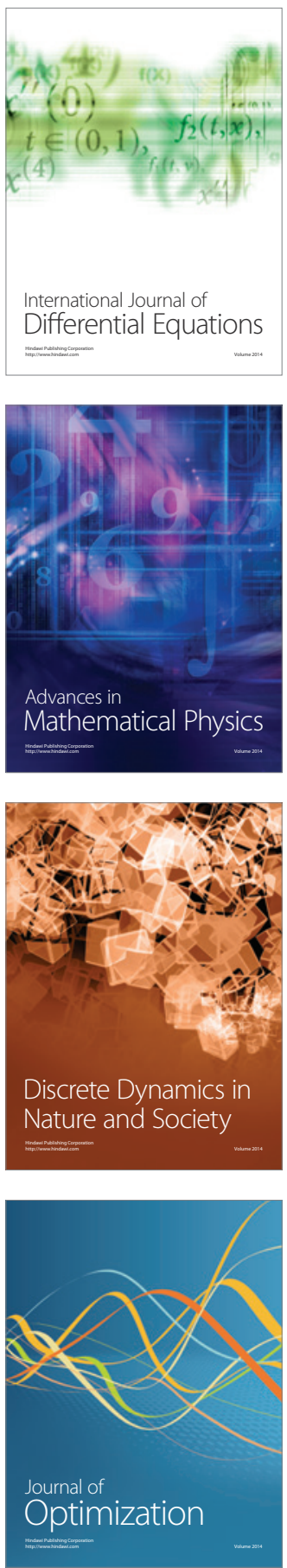